\title{
Frecuencia de estreñimiento en pacientes de Atención Primaria
}

\author{
$M^{a}$ Soledad Navarro Ruiza , Carmen Llanos Val Jiméneza, Eva García Atienza ${ }^{a}$, \\ Lorena Moreno de la Rosa ${ }^{a}$, María Flores Copete ${ }^{a}$, Katia Chávez Tafura y \\ Jaime López-Torres López ${ }^{\text {b }}$
}

\begin{abstract}
${ }^{a}$ MIR de Medicina Familiar y Comunitaria. Gerencia de Atención Integrada de Albacete. Albacete (España). ${ }^{b}$ MIR de Anestesiología y Reanimación. Hospital Universitari y Politècnic "La Fe". Valencia (España).

Correspondencia: $\mathrm{M}^{\mathrm{a}}$ Soledad Navarro Ruiz. C/ Alameda, 5. C.P. 16220. Quintanar del Rey - Cuenca (España). Correo electrónico: soledadnavarroruiz@gmail.com

Recibido el 8 de septiembre de 2014.

Aceptado para su publicación el 1 de octubre de 2014.
\end{abstract}

\begin{abstract}
RESUMEN
Objetivo: Determinar con qué frecuencia presentan estreñimiento los pacientes que acuden por cualquier motivo a la consulta del médico de familia y comprobar su asociación con las características sociodemográficas, la existencia de enfermedades digestivas diagnosticadas previamente y el consumo de fármacos.
\end{abstract}

Diseño del estudio: Estudio de prevalencia y asociación cruzada.

Emplazamiento: Atención Primaria.

Participantes: Una muestra de 473 pacientes seleccionados mediante muestreo sistemático en cuatro consultas de medicina de familia.

Mediciones principales: Presencia de síntomas de estreñimiento evaluado mediante la escala GSRS (Gastrointestinal Symptom Rating Scale). Otras variables: diagnóstico previo de enfermedades del aparato digestivo, consumo de medicación, frecuentación y características sociodemográficas.

Resultados: El 32,0\% de los pacientes (IC 95\%: 27,5 - 36,5) declararon la presencia de síntomas de estreñimiento con intensidad moderada o fuerte: disminución de la frecuencia en el número de deposiciones, aumento en la consistencia de las heces o sensación de evacuación incompleta. Los síntomas fueron más frecuentes en mujeres $(37,3 \%$ vs. $24,0 \% ; p=0,004)$ y en consumidores de fármacos para enfermedades del aparato digestivo $(47,7 \%$ vs. $23,8 \%$; $p$ $<0,001)$. Se observó una tendencia lineal, estadísticamente significativa $(p<0,001)$, entre la presencia de estreñimiento y el número total de fármacos consumidos por los pacientes.

Conclusiones: Es elevada la frecuencia de síntomas de estreñimiento en los pacientes de Atención Primaria, especialmente en el sexo femenino. Se observa una relación lineal entre la frecuencia de estos síntomas y el consumo de fármacos, por lo que el estreñimiento debe ser considerado como un potencial efecto adverso frecuente en pacientes polimedicados.

PALABRAS CLAVE: Estreñimiento. Atención Primaria de Salud. Signos y Síntomas Digestivos. ABSTRACT

Frequency of constipation in primary care patients

Objective: To determine how often patients attending family physician's consultation for whatever reason present constipation, and to verify its association with sociodemographic characteristics, existence of previously diagnosed digestive diseases and drug consumption.

Study design: Prevalence study and crossed association.

Setting: Primary health care.

Participants: A sample of 473 patients selected by systematic sampling in four family medicine consultations.

Measurements: Presence of symptoms of constipation assessed by the GSRS scale (Gastrointestinal Symptom Rating Scale). Other variables: previous diagnosis of gastrointestinal tract diseases, medication use, frequency of attendance, and sociodemographic characteristics.

Results: $32,0 \%$ of the patients (IC $95 \%: 27,5-36,5)$ stated the presence of symptoms of constipation with moderate or strong intensity: decrease in the frequency of the number of bowel movements, increase in the consistency of the faeces, or sensation of incomplete evacuation. The symptoms were more frequent in women $(37,3 \%$ vs. $24,0 \% ; p=0,004)$ and in consumers of medication for diseases of the digestive system $(47,7 \%$ vs. $23,8 \% ; p<0,001)$. A linear statistically significant trend was observed $(p<0,001)$, between the presence of constipation and the total number of drugs used by the patients.

Conclusions: The frequency of symptoms of constipation is high in Primary health care patients, especially in females. A linear relationship between the frequency of these symptoms and drug consumption is observed, whereby constipation should be regarded as a common potential side effect in patients with polypharmacy. 


\section{INTRODUCCIÓN}

El estreñimiento es un problema crónico en muchos pacientes y, en ocasiones, como ocurre en ancianos, constituye un problema sanitario importante; sin embargo, en la mayoría de los casos es un motivo de consulta que provoca molestias pero no incapacita al paciente.

La palabra "estreñimiento" puede tener un significado diferente para pacientes y profesionales sanitarios, incluso entre diferentes culturas. Se define mediante síntomas intestinales, como paso difícil o poco frecuente de heces, dureza de las mismas o sensación de evacuación incompleta, y puede ocurrir de manera aislada o ser secundario a otro trastorno subyacente. Muchos autores consideran estreñimiento como sinónimo de frecuencia reducida en la defecación, aunque otros consideran también esfuerzo para defecar, heces duras e incapacidad para la defección. Al respecto, los criterios modificados de Roma III incorporan, como criterios generales, una duración de al menos 3 meses durante un periodo de 6 , síntomas específicos al menos en una de cada 4 defecaciones, criterios insuficientes de síndrome de intestino irritable e inexistencia de deposiciones o deposiciones escasas disminuidas de consistencia ${ }^{1}$. Como criterios específicos se incluyen esfuerzo para defecar, materias fecales grumosas o duras, sensación de evacuación incompleta, bloqueo u obstrucción anorrectal y recurrencia a maniobras manuales o digitales para facilitar la defecación.

El diagnóstico de los trastornos fecales también requiere resultados alterados en pruebas anorrectales $^{2}$, pero por lo general estos criterios sólo se utilizan en investigación, son poco útiles en la práctica clínica diaria y son poco concordantes con la opinión del paciente p $^{3-4}$.

En una revisión sistemática publicada en 2011 sobre epidemiología del estreñimiento ${ }^{5}$, en la que se identificaron 58 estudios sobre prevalencia de estreñimiento en la población, tanto en niños como en adultos, se observaron diferencias en las definiciones de estreñimiento, señalándose que los datos obtenidos mediante cuestionarios podrían incluir causas orgánicas de estreñimiento. A pesar de estas limitaciones, la prevalencia de estreñimiento sería del $16,0 \%$ en adultos en general y del $33,5 \%$ en adultos mayores de 60 años. Aunque no todos, la mayoría de los estudios sugieren que la prevalencia de estreñimiento es mayor en población blanca, en mujeres (relación 1,5:1) y en individuos institucionalizados. Por otra parte, las mujeres también son más propensas a usar laxantes y a buscar atención médica para su estreñimiento. La prevalencia en la población europea varía entre el $2 \%$ y el $27 \%^{3}$, y en España la prevalencia autodeclarada es de un $29,5 \%{ }^{4}$.

Existe acuerdo en cuanto a los factores de riesgo para el estreñimiento, incluyéndose un bajo nivel socioeconómico, un estilo de vida sedentaria, el uso de medicamentos, el embarazo y enfermedades que alteran la motilidad gastrointestinal, tales como la enfermedad por reflujo gastroesofágico, el síndrome de intestino irritable y la dispepsia funcional $^{6-7}$. Aunque sólo una minoría de los pacientes afectados busca atención médica para el tratamiento, en las consultas de Atención Primaria el estreñimiento ha llegado a ser calificado como uno de los 5 diagnósticos más frecuentes ${ }^{8}$.

El objetivo del estudio ha sido determinar con qué frecuencia presentan estreñimiento los pacientes que acuden por cualquier motivo a la consulta del médico de familia y comprobar su asociación con las características sociodemográficas, la existencia de enfermedades digestivas diagnosticadas previamente y el consumo de fármacos.

\section{SUJETOS Y MÉTODOS}

Se trata de un estudio observacional y descriptivo de prevalencia y asociación cruzada realizado en Atención Primaria. Para ello se seleccionaron 473 pacientes mediante muestreo sistemático (constante de muestreo: uno de cada cuatro pacientes) en cuatro consultas de medicina de familia, pertenecientes al Centro de Salud Zona IV de la ciudad de Albacete. Dicho tamaño muestral corresponde a una frecuencia esperada de estreñimiento del $24 \%$, un nivel de confianza del 95\%, una precisión de $\pm 4 \%$ y una previsión de no respuestas del $10 \%$. El criterio de inclusión fue acudir a la consulta del médico de familia por cualquier motivo, tanto por problemas de salud como por razones burocráticas. Fueron excluidos los pacientes que, una vez informados del objetivo del estudio y solicitado su consentimiento, rechazaron la participación. Los datos necesarios para el estudio fueron recogidos durante el periodo comprendido entre octubre y diciembre de 2012.

Los sujetos que aceptaron participar fueron evaluados a través de un cuestionario que incluyó las preguntas sobre estreñimiento contenidas en la escala GSRS (Gastrointestinal Symptom Rating Scale), la cual constituye un instrumento específico bien documentado para evaluar la frecuencia de síntomas gastrointestinales ${ }^{9}$.Dicho instrumento ha sido traducido y validado en población española y presenta unos adecuados índices psicométricos. Las preguntas sobre estreñimiento se refieren a la existencia del mismo durante la última semana, así como a la presencia de heces duras y a la sensación de evacuación incompleta. Se trata de una escala tipo Likert de 7 grados, donde el 1 representa la opción más positiva y el 7 la más negativa. Siguiendo las recomendaciones de quienes diseñaron la escala, cuando uno de los ítems no fue respondido se le asignó la puntuación media del resto.

Otras variables incluidas en el cuestionario fueron las relacionadas con las características sociodemográficas de los pacientes: edad, sexo y nivel de estudios. Posteriormente, a partir de la historia clínica informatizada se obtuvieron los siguientes datos: diagnóstico previo de enfermedades del aparato digestivo (Clasificación Internacional en Atención Primaria), fármacos para dichas enfermedades (Clasificación Anatómico-Terapéutica), número total de fármacos y número de visitas al 
médico de familia durante los 3 meses previos a la entrevista.

La información fue introducida en una base de datos y analizada posteriormente mediante el programa estadístico IBM SPSS v.19. La estrategia de análisis consistió en una descripción de las variables de estudio mediante proporciones y construcción de intervalos de confianza al 95\%, así como medidas de tendencia central y dispersión según la naturaleza de las variables. Así mismo, se llevó a cabo un análisis comparativo entre sujetos con o sin estreñimiento a través de pruebas de comparación proporciones (Chi-cuadrado y tendencia lineal de Mantel-Haenszel) en grupos independien- tes, utilizando un nivel de significación de $p<0,05$. Finalmente, las variables asociadas fueron contrastadas mediante un modelo de regresión logística cuya variable dependiente fue la presencia de estreñimiento.

\section{RESULTADOS}

De los 473 pacientes seleccionados, respondieron 435 , siendo la tasa de respuesta del $92,0 \%$. Su edad media fue de 48,0 años (DE: 17,7) y la proporción de mujeres fue del $59,8 \%$. El resto de las características de los pacientes, tanto clínicas como sociodemográficas, se muestran en la tabla 1. Las

\begin{tabular}{|c|c|c|c|}
\hline Características & $\mathbf{N}^{\circ}(\%)$ & $\begin{array}{l}\text { \% Estreñimiento } \\
\text { (IC 95\%) }\end{array}$ & $\mathbf{p}$ \\
\hline \multicolumn{4}{|l|}{ Sexo } \\
\hline Hombre & $175(40,2)$ & $24,0(17,4-30,6)$ & \multirow{2}{*}{0,004} \\
\hline Mujer & $260(59,8)$ & $37,3(31,2-43-4)$ & \\
\hline \multicolumn{4}{|l|}{ Edad } \\
\hline Menos de 65 años & $351(80,7)$ & $29,1(24,2-34,0)$ & \multirow{2}{*}{0,008} \\
\hline 65 años o más & $84(19,3)$ & $44,0(32,8-55,3)$ & \\
\hline \multicolumn{4}{|l|}{ Nivel de estudios } \\
\hline Sin estudios & $58(13,3)$ & $37,9(24,6-51,3)$ & \multirow{4}{*}{ NS } \\
\hline Estudios primarios & $209(48,0)$ & $32,5(25,9-39,1)$ & \\
\hline Enseñanza secundaria & $96(22,1)$ & $22,9(14,0-31,8)$ & \\
\hline Estudios universitarios & $72(16,6)$ & $37,5(25,6-49,4)$ & \\
\hline \multicolumn{4}{|c|}{ Diagnóstico previo de enfermedades digestivas } \\
\hline Sí & $114(26,2)$ & $40,4(30,9-49,8)$ & \multirow{2}{*}{0,02} \\
\hline No & $321(73,8)$ & $29,0(23,9-34,1)$ & \\
\hline \multicolumn{4}{|c|}{ Consumo de fármacos para enfermedades digestivas } \\
\hline Sí & $149(34,3)$ & $47,7(39,3-56,0)$ & \multirow{2}{*}{$<0,001$} \\
\hline No & $286(65,7)$ & $23,8(18,7-28,9)$ & \\
\hline \multicolumn{4}{|c|}{ Consumo de cualquier fármaco } \\
\hline Ningún fármaco & $133(30,6)$ & $18,8(11,1-25,0)$ & \multirow{3}{*}{$<0,001$} \\
\hline 1 a 3 & $157(36,1)$ & $36,9(29,1-44,8)$ & \\
\hline 4 o más & $145(33,3)$ & $39,3(31,0-47,6)$ & \\
\hline \multicolumn{4}{|c|}{ Visita al médico de familia durante los 3 meses previos } \\
\hline 1 a 3 & $299(68,7)$ & $27,4(22,2-32,6)$ & \multirow{2}{*}{0,003} \\
\hline 4 o más & $136(31,3)$ & $41,9(33,3-50,6)$ & \\
\hline
\end{tabular}

NS: Diferencia estadísticamente no significativa

Tabla 1. Características clínicas y sociodemográficas. 


\begin{tabular}{|c|c|}
\hline Síntomas y enfermedades del aparato digestivo & $N^{\circ}(\%)$ \\
\hline Dolor de estómago / epigástrico & $1(0,2)$ \\
\hline Pirosis & $2(0,5)$ \\
\hline Dispepsia / indigestión & $15(3,4)$ \\
\hline Flatulencia / aerofagia / gases & $1(0,2)$ \\
\hline Vómito & $1(0,2)$ \\
\hline Estreñimiento & $9(2,0)$ \\
\hline Hematemesis / vómitos de sangre & $2(0,5)$ \\
\hline Rectorragia / hemorragia rectal & $1(0,2)$ \\
\hline Infección gastrointestinal & $13(3,0)$ \\
\hline Hepatitis viral & $6(1,4)$ \\
\hline Neoplasias malignas de colon / recto & $4(0,9)$ \\
\hline Neoplasias benignas / inespecíficas de aparato digestivo & $1(0,2)$ \\
\hline Otra lesiones de aparato digestivo & $14(3,2)$ \\
\hline Enfermedades del esófago & $20(4,6)$ \\
\hline Úlcera duodenal & $3(0,7)$ \\
\hline Otras úlceras pépticas & $7(1,6)$ \\
\hline Hernia inguinal & $4(0,9)$ \\
\hline Hernia de hiato & $13(3,0)$ \\
\hline Otras hernias abdominales & $2(0,5)$ \\
\hline Enfermedad diverticular del intestino & $5(1,1)$ \\
\hline Síndrome de colon irritable & $7(1,6)$ \\
\hline Enteritis crónica / colitis ulcerosa & $5(1,1)$ \\
\hline Fisura anal / absceso anal & $5(1,1)$ \\
\hline Enfermedades hepáticas NE & $14(3,2)$ \\
\hline Colecistitis / colelitiasis & $6(1,4)$ \\
\hline Otras enfermedades del aparato digestivo & $4(0,9)$ \\
\hline
\end{tabular}

Tabla 2. Distribución de síntomas y enfermedades del aparato digestivo (Clasificación Internacional de la Atención Primaria, CIAP 2).

enfermedades del aparato digestivo diagnosticadas previamente aparecen en la tabla 2.

Consumían fármacos para el aparato digestivo 149 pacientes $(34,3 \%)$, siendo los más frecuentes los inhibidores de la bomba de protones, los cuales eran consumidos por el 30,3\%. A continuación, los grupos más consumidos eran procinéticos $(2,5 \%)$, antagonistas $\mathrm{H}-2(2,1 \%)$, espasmolíticos $(1,8 \%)$, otros antiácidos $(1,2 \%)$, laxantes $(0,9 \%)$ y otros $(0,9 \%)$. Eran consumidores de cualquier fármaco el $69,4 \%$, siendo el número medio de fármacos entre los consumidores de 4,3 (DE: 3,1).

En cuanto a la presencia de estreñimiento, manifestaron una disminución de la frecuencia en el número de deposiciones durante la última semana, al menos con intensidad moderada, el 19,8\% de los pacientes, un aumento en la consistencia de las heces durante el mismo periodo el $15,8 \%$ y sensación de evacuación incompleta el 21,9\% (figura 1 y tabla 3). En conjunto, el 32,0\% de los pacientes (IC 95\%: 27,5 - 36,5) declararon la presencia de cualquiera de estos síntomas con intensidad moderada o fuerte durante los días previos a la entrevista.

La presencia de síntomas de estreñimiento fue significativamente más frecuente en mujeres $(37,3 \%$ vs. $24,0 \% ; p=0,004)$, en mayores de 65 años $(44,0 \%$ vs. $29,1 \% ; p=0,008)$, en pacientes diagnosticados previamente de alguna enfermedad del aparato digestivo $(40,4 \%$ vs. $29,0 \% ; p=0,02)$ y en consumidores de fármacos para estas enfermedades (47,7\% vs. 23,8\%; p<0,001). Además, se observó una tendencia lineal, estadísticamente significativa $(p<0,001)$, entre la presencia de estreñimiento y el 


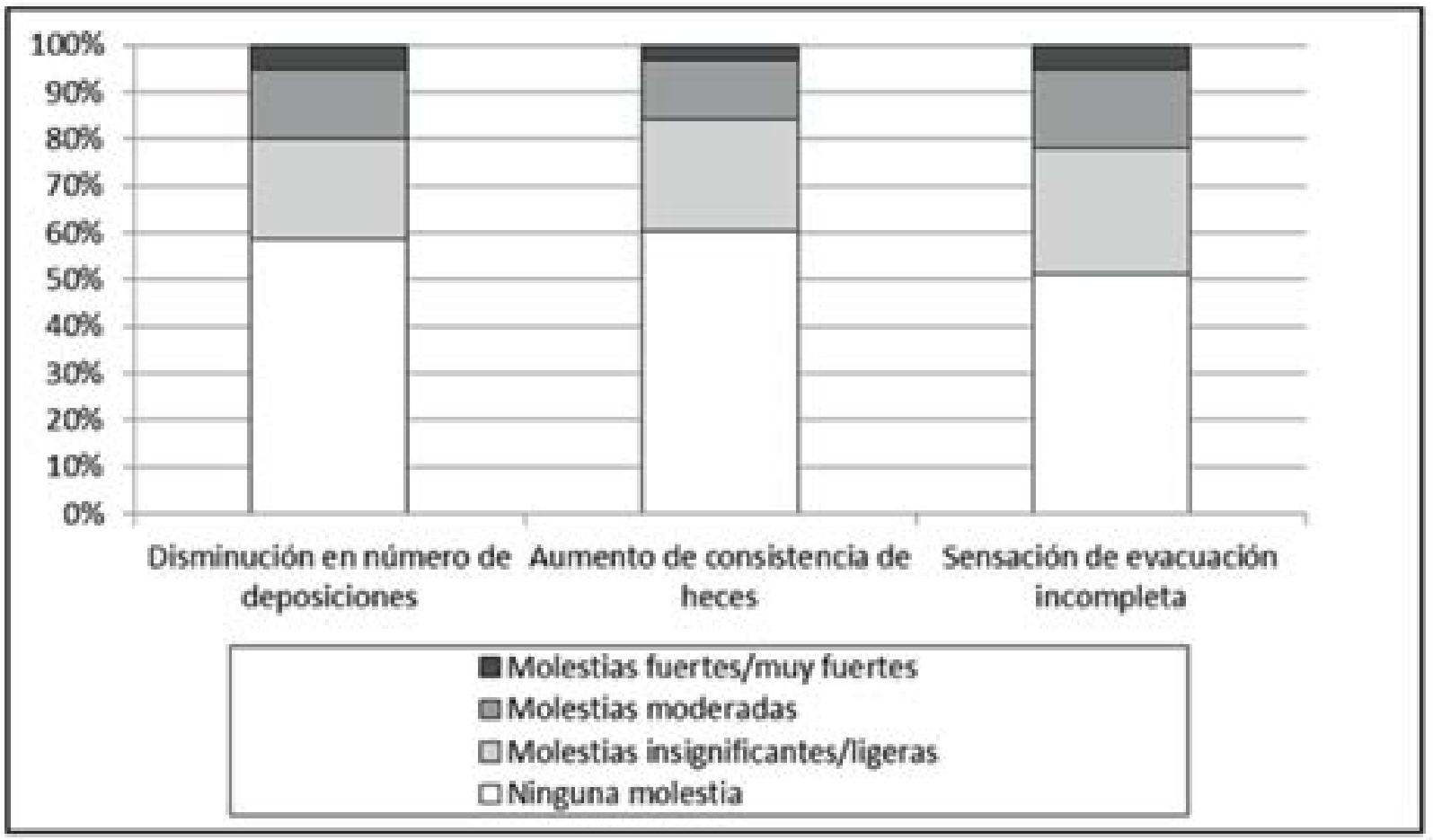

Figura 1. Distribución de los pacientes según la presencia de síntomas de estreñimiento.

número de fármacos totales consumidos por los pacientes: $18,0 \%$ en no consumidores, $36,9 \%$ en consumidores de 1 a 3 fármacos y $39,3 \%$ en consumidores de 4 o más.
Mediante regresión logística se comprobó que las variables asociadas de forma independiente con la presencia de estreñimiento (tabla 4) fueron sexo femenino (OR: 2,1), consumo de fármacos para las enfermedades del aparato digestivo $(\mathrm{OR}: 2,4)$ y

\begin{tabular}{|c|c|c|c|c|c|c|}
\hline \multirow{2}{*}{ Características } & \multicolumn{2}{|c|}{ Hombres } & \multicolumn{2}{|c|}{ Mujeres } & \multicolumn{2}{|c|}{ Total } \\
\hline & $\mathrm{N}^{\circ}(\%)$ & IC $95 \%$ & $\mathbf{N}^{\circ}(\%)$ & IC $95 \%$ & $\mathbf{N}^{\circ}(\%)$ & IC $95 \%$ \\
\hline \multicolumn{7}{|l|}{$\begin{array}{l}\text { Disminución en número de } \\
\text { deposiciones }\end{array}$} \\
\hline Sin molestias / molestias ligeras & $153(87,4)$ & $82,2-92,6$ & $196(75,4)$ & $70,0-80,8$ & $349(80,2)$ & $76,4-84,1$ \\
\hline Molestias moderadas / fuertes & $22(12,6)$ & $7,4-17,8$ & $64(24,6)$ & $19,2-30,0$ & $86(19,8)$ & $15,9-23,6$ \\
\hline \multicolumn{7}{|l|}{$\begin{array}{l}\text { Aumento de consistencia de } \\
\text { heces }\end{array}$} \\
\hline Sin molestias / molestias ligeras & $155(88,6)$ & $83,6-93,6$ & $211(81,2)$ & $76,2-86,1$ & $366(84,1)$ & $80,6-87,7$ \\
\hline Molestias moderadas / fuertes & $20(11,4)$ & $6,4-16,4$ & $49(18,8)$ & $13,9-23,8$ & $69(15,9)$ & $12,3-19,4$ \\
\hline \multicolumn{7}{|l|}{$\begin{array}{l}\text { Sensación de evacuación } \\
\text { incompleta }\end{array}$} \\
\hline Sin molestias / molestias ligeras & $141(80,6)$ & $74,4-86,7$ & $199(76,5)$ & $71,2-81,9$ & $340(78,2)$ & $74,2-82,2$ \\
\hline Molestias moderadas / fuertes & $34(19,4)$ & $13,3-25,6$ & $61(23,5)$ & $18,1-28,8$ & $95(21,8)$ & $17,8-25,8$ \\
\hline
\end{tabular}

Tabla 3. Distribución de los síntomas de estreñimiento en hombres y mujeres. 


\begin{tabular}{|c|c|c|c|c|c|c|}
\hline Variables & B & $\begin{array}{c}\text { Error } \\
\text { estándar }\end{array}$ & Wald & $\mathbf{p}$ & OR & IC 95\% \\
\hline Sexo femenino & 0,794 & 0,232 & 11,688 & 0,001 & 2,2 & $1,4-3,5$ \\
\hline $\begin{array}{l}\text { Consumo de fármacos para } \\
\text { enfermedades del aparato digestivo }\end{array}$ & 0,840 & 0,242 & 12,081 & 0,001 & 2,3 & $1,4-3,7$ \\
\hline $\begin{array}{l}\text { Consumo de } 2 \text { o más fármacos para } \\
\text { cualquier enfermedad }\end{array}$ & 0,520 & 0,253 & 4,217 & 0,040 & 1,7 & $1,1-2,8$ \\
\hline Edad superior a 65 años & 0,315 & 0,285 & 1,229 & 0,268 & 1,4 & $0,8-2,4$ \\
\hline
\end{tabular}

Tabla 4. Variables asociadas mediante regresión logística a la presencia de estreñimiento.

consumo de 2 o más fármacos para cualquier enfermedad (OR: 1,8).

\section{DISCUSIÓN}

En nuestros resultados hemos observado que aproximadamente un tercio de los pacientes que acuden por cualquier motivo a las consultas de Atención Primaria consumen fármacos para el aparato digestivo, de los cuales los más utilizados son los inhibidores de la bomba de protones. También comprobamos que el consumo de cualquier medicación es frecuente en los pacientes mayores de 18 años, ya que más de la mitad de ellos utilizan fármacos, siendo el número medio de 4 . En cuanto a la presencia de estreñimiento, en la muestra estudiada constituye un aspecto clínico importante, pues un tercio de los participantes presentan síntomas de intensidad moderada o fuerte durante la semana previa a la entrevista. Este hallazgo es más frecuente en mujeres, en mayores de 65 años, en pacientes con enfermedades digestivas y en consumidores de fármacos para estas enfermedades, constatándose una relación lineal entre la presencia de síntomas de estreñimiento y el número de fármacos consumidos.

En una revisión publicada en octubre de 2010, donde se analizan 58 artículos sobre prevalencia de estreñimiento ${ }^{5}$, se concluye que el $16,0 \%$ de los adultos en la población general presenta estreñimiento, ascendiendo al $33,5 \%$ en los mayores de 60 años. Puesto que nuestros resultados se refieren a pacientes, es comprensible que sean superiores las cifras de prevalencia obtenidas. Si bien en dicha revisión, al igual que es observado en nuestro estudio, la frecuencia del estreñimiento es superior en mujeres y en mayores de 65 años, no se menciona, sin embargo, la relación de estos síntomas con el consumo de fármacos, lo cual ha sido objetivado en nuestros resultados. Un estudio publicado recientemente en Francia revela que una de cada 4 ó 5 personas $(22,4 \%)$ presenta estreñimiento, motivando hasta en un $66 \%$ de ellas un empeoramiento de la calidad de vida ${ }^{10}$. En población española un estudio publicado en 2004 señala cifras de prevalencia de estreñimiento autoproclamado del 29,5\%, reduciéndose esta cifra hasta el $14 \%$ cuando se siguen los criterios de Roma $\mathrm{II}^{4}$. Los diferentes criterios utilizados condicionan ampliamente la variabilidad de los resultados a la hora de establecer la frecuencia de estreñimiento, lo cual dificulta enormemente su comparación. Nuestros resultados han sido obtenidos mediante la escala de síntomas gastrointestinales GSRS, pero no conocemos la existencia de estudios previos realizados en población española que hayan utilizado el mismo instrumento de evaluación, motivo por lo que no es posible efectuar la comparación de los datos obtenidos.

Como limitación a los resultados, cabe señalar la exclusión de la población no consultante, lo cual ha podido contribuir a incrementar las cifras de prevalencia obtenidas. Es muy probable que la frecuencia de estreñimiento sea sensiblemente inferior al considerar el estudio de la población general y, en consecuencia, que sea reducida la validez externa de nuestros resultados. Sin embargo, hay que tener en cuenta que se ha incluido en la muestra a personas que consultaron por cualquier motivo durante el periodo en el que fueron recogidos los datos, resultando infrecuente el estreñimiento como motivo de consulta, especialmente si consideramos que la terapia farmacológica correspondiente carece de financiación por parte de nuestro sistema sanitario. Por otra parte, la aproximación al estudio de los síntomas está limitada por la repercusión de éstos en los sujetos que los padecen y la diferente percepción de enfermedad que pueden conllevar.

En futuras investigaciones sería deseable una mayor uniformidad en cuanto a los criterios utilizados para juzgar la presencia de estreñimiento y, por otra parte, resultará del máximo interés evaluar en el ámbito de Atención Primaria la efectividad de intervenciones dirigidas a modificar estilos de vida a través de recomendaciones sobre dieta y ejercicio físico, cuyos beneficios parecen indudables. Así mismo, la información sobre utilización racional de los fármacos para combatir el estreñimiento, en caso necesario, debería ser una prioridad para los profesionales sanitarios, dado que se trata de un síntoma muy frecuente.

En conclusión, es elevada la frecuencia de síntomas de estreñimiento en los pacientes de Atención Primaria, especialmente en el sexo femenino. Además, se observa una relación lineal entre la frecuencia de estos síntomas y el consumo de fármacos, por lo que el estreñimiento debe ser considerado como un potencial efecto adverso frecuente en pacientes polimedicados. 


\section{BIBLIOGRAFÍA}

1. Longstreth GF, Thompson WG, Chey WD, Houghton LA, Mearin F, Spiller RC. Functional bowel disorders. Gastroenterology. 2006; 130 (5): 1480-91.

2. Bharucha $A E$, Wald $A$, Enck $P$, Rao $S$. Functional anorectal disorders. Gastroenterology. 2006; 130 (5): 1510-8.

3. Tack J, Müller-Lissner S, Stanghellini V, Boeckxstaens G, Kamm MA, Simren $M$ et al. Diagnosis and treatment of chronic constipation - a European perspective. Neurogastroenterol Motil. 2011; 23 (8): 697-710.

4. Garrigues V, Gálvez C, Ortiz V, Ponce M, Nos P, Ponce J. Prevalence of constipation: agreement among several criteria and evaluation of the diagnostic accuracy of qualifying symptoms and self-reported definition in a population-based survey in Spain. Am J Epidemiol. 2004; 159 (5): 520-6.

5. Mugie SM, Benninga MA, Di Lorenzo C. Epidemiology of constipation in children and adults: a systematic review. Best Pract Res Clin Gastroenterol. 2011; 25 (1): 3-18.
6. Higgins PD, Johanson JF. Epidemiology of constipation in North America: a systematic review. Am J Gastroenterol. 2004; 99 (4): 750-9.

7. Locke GR, Zinsmeister AR, Fett SL, Melton LJ, Talley NJ. Overlap of gastrointestinal symptom complexes in a US community. Neurogastroenterol Motil. 2005; 17 (1): 29-34.

8. Bharucha AE, Pemberton JH, Locke GR. American Gastroenterological Association technical review on constipation. Gastroenterology. 2013; 144 (1): 218-38.

9. Svedlund J, Sjödin I, Dotevall G. GSRS--a clinical rating scale for gastrointestinal symptoms in patients with irritable bowel syndrome and peptic ulcer disease. Dig Dis Sci. 1988; 33 (2): 129-34.

10. Siproudhis L, Pigot F, Godeberge P, Damon H, Soudan D, Bigard MA. Defecation disorders: a French population survey. Dis Colon Rectum. 2006; 49 (2): 219-27. 